\title{
Regression of Human Prostate Tumors and Metastases in Nude Mice following Treatment with the Recombinant Oncolytic Vaccinia Virus GLV-1h68
}

\author{
Ivaylo Gentschev, ${ }^{1,2}$ Ulrike Donat, ${ }^{2}$ Elisabeth Hofmann, ${ }^{2}$ Stephanie Weibel, ${ }^{2}$ \\ Marion Adelfinger, ${ }^{2}$ Viktoria Raab, ${ }^{2}$ Martin Heisig, ${ }^{3}$ Nanhai Chen, ${ }^{1}$ Yong A. Yu, \\ Jochen Stritzker, ${ }^{1,2}$ and Aladar A. Szalay ${ }^{1,2,4,5,6}$ \\ ${ }^{1}$ Genelux Corporation, San Diego Science Center, San Diego, CA 92109, USA \\ ${ }^{2}$ Department of Biochemistry, University of Wuerzburg, D-97074 Wuerzburg, Germany \\ ${ }^{3}$ Institute for Medical Radiation and Cell Research, University of Wuerzburg, D-97078 Wuerzburg, Germany \\ ${ }^{4}$ Rudolf Virchow Center for Experimental Biomedicine, University of Wuerzburg, D-97078 Wuerzburg, Germany \\ ${ }^{5}$ Institute for Molecular Infection Biology, University of Wuerzburg, D-97078 Wuerzburg, Germany \\ ${ }^{6}$ Department of Radiation Oncology, Moores Cancer Center, University of California, San Diego, 3855 Health Sciences Drive 0843, \\ La Jolla, CA 92093-0843, USA
}

Correspondence should be addressed to Aladar A. Szalay, aaszalay@genelux.com

Received 17 July 2009; Revised 14 October 2009; Accepted 13 January 2010

Academic Editor: Colin Cooper

Copyright ( 2010 Ivaylo Gentschev et al. This is an open access article distributed under the Creative Commons Attribution License, which permits unrestricted use, distribution, and reproduction in any medium, provided the original work is properly cited.

\begin{abstract}
Virotherapy using oncolytic vaccinia virus strains is one of the most promising new strategies for cancer therapy. In the current study, we analyzed the therapeutic efficacy of the oncolytic vaccinia virus GLV-1h68 against two human prostate cancer cell lines DU-145 and PC-3 in cell culture and in tumor xenograft models. By viral proliferation assays and cell survival tests, we demonstrated that GLV-1h68 was able to infect, replicate in, and lyse these prostate cancer cells in culture. In DU-145 and PC-3 tumor xenograft models, a single intravenous injection with GLV-1h68 resulted in a significant reduction of primary tumor size. In addition, the GLV-1h68-infection led to strong inflammatory and oncolytic effects resulting in drastic reduction of regional lymph nodes with PC-3 metastases. Our data documented that the GLV-1h68 virus has a great potential for treatment of human prostate carcinoma.
\end{abstract}

\section{Introduction}

Despite some progress in the diagnosis and treatment of prostate cancer $(\mathrm{PCa})$, this disease remains the second leading cause of cancer-related death in men. The disease accounts for an estimated 27,050 deaths in 2007 in the USA alone [1]. Death of prostate cancer patients is normally caused by the formation of metastases [2,3]. At the present time, there is no effective treatment for the inhibition of PCa metastases. Therefore, the development of new therapies and diagnostics for $\mathrm{PCa}$ metastases is a high priority. One of the most promising novel cancer therapies for humans is oncolytic virotherapy. The concept that viruses may be useful for eradication of cancer has already been confirmed by the use of several viruses, for example, Newcastle disease virus, reovirus, lentivirus, herpes simplex virus, enterovirus, Sindbis virus, Semliki Forest virus, Seneca Valley virus, and vaccinia virus [4-7].

In our study, we have investigated the therapeutic potential of the oncolytic vaccinia virus GLV-1h68 in xenograft models of human prostate cancer. The GLV-1h68 virus was engineered by inserting expression cassettes encoding a Renilla luciferase-green fluorescent protein (Ruc-GFP) fusion protein, $\beta$-galactosidase, and $\beta$-glucuronidase into the genome of the LIVP strain, which is highly attenuated compared to the wild-type strain [8]. We have already demonstrated that the injection of GLV-1h68 leads to regression and elimination of different tumor xenografts in 
nude mice [8-13]. In addition, Kelly et al. reported that GLV1 h68 virus could be used as a tool for detection of melanoma lymph node metastases in an immunocompetent animal model [14]. More recently, a GLV-1h68 derivative (GLV1h99) that expresses the human norepinephrine transporter was shown to be useful for both therapy and deep-tissue imaging of tumors $[15,16]$. In the meantime, more and more data reported from clinical trials using oncolytic vaccinia viruses in cancer patients have shown good safety and promising responses $[7,17]$.

Here we describe that GLV-1h68 was able to infect, replicate in, and lyse human prostate DU-145 and PC-3 cells in culture. In addition, a single injection of GLV-1h68 into mice with prostate tumor xenografts could efficiently prevent tumor growth and formation of PC3-metastases in the local lymph nodes. Finally, the oncolytic and immunological effects of GLV-1h68 in primary tumors and PC-3 metastases were analyzed by fluorescence imaging, immunohistochemistry, and an Immune-Related Protein Antigen Profiling.

\section{Materials and Methods}

2.1. Cell Culture. African green monkey kidney fibroblasts (CV-1) and human prostate cancer cell lines (DU-145 and PC-3) were obtained from the American Type Culture Collection (ATCC). Cells were cultured in DMEM supplemented with antibiotic-solution (100 units/mL penicillin G, 100 units/mL streptomycin) and 10\% fetal bovine serum (FBS; Invitrogen $\mathrm{GmbH}$, Karlsruhe, Germany) at $37^{\circ} \mathrm{C}$ under $5 \%$ $\mathrm{CO}_{2}$.

2.2. Virus Strain. GLV-1h68 is a genetically stable oncolytic virus strain designed to locate, enter, colonize, and destroy cancer cells without harming healthy tissues or organs [8].

2.3. Cell Viability Assay with GLV-1h68. DU-145 and PC3 cells were seeded onto 24-well plates (Nunc, Wiesbaden, Germany). After 24 hours in culture, cells were infected with GLV-1h68 using multiplicities of infection (MOI) of 0.1 and 1 . Cells were incubated at $37^{\circ} \mathrm{C}$ for 1 hour, then the infection medium was removed and the cells were incubated in fresh growth medium. The amount of viable cells after infection with GLV-1h68 was measured using 3-(4, 5-dimethylthiazol-2-yl)-2, 5-diphenyltetrazolium bromide (MTT) (Sigma, Taufkirchen, Germany). At 24, 48, 72, or 96 hours after infection of cells, medium was replaced by $0.5 \mathrm{~mL}$ MTT solution at a concentration of $2.5 \mathrm{mg} / \mathrm{mL}$ MTT dissolved in RPMI 1640 without phenol red and incubated for 2 hours at $37^{\circ} \mathrm{C}$ in a $5 \% \mathrm{CO}_{2}$ atmosphere. After removal of the MTT solution, the color reaction was stopped by adding $1 \mathrm{~N} \mathrm{HCl}$ diluted in isopropanol. The optical density was then measured at a wavelength of $570 \mathrm{~nm}$. Uninfected cells were used as reference and were considered as 100\% viable. The amount of viable cells after infection with GLV$1 \mathrm{~h} 68$ was measured in triplicate.

2.4. Viral Replication. DU-145 and PC-3 cells grown in 24well plates were infected with GLV-1h68 at an MOI of 0.1 .
After one hour of incubation at $37^{\circ} \mathrm{C}$ with gentle agitation every 20 minutes, the infection medium was removed and replaced by fresh growth medium. Supernatants were collected from virally treated cells at 1, 6, 12, 24, 48, 72, or 96 hours postinfection. Serial dilutions of supernatants were titrated by standard plaque assays on CV-1 cells. All samples were measured in triplicate.

2.5. GLV-1h68 Mediated Therapy of DU-145 and PC-3 Xenografts. Tumors were generated by implanting DU-145 $\left(1 \times 10^{7}\right.$ cells in $100 \mu L$ of PBS $)$ or PC-3 cells $\left(2.5 \times 10^{6}\right.$ in $100 \mu \mathrm{L}$ PBS) subcutaneously on the right flank above the hind leg of 6- to 8-week-old male or female nude mice (NCI/Hsd/Athymic Nude-Foxn $1^{\text {nu}}$, Harlan Winkelmann $\mathrm{GmbH}$, Borchen, Germany). Tumor growth was recorded weekly in two dimensions using a digital caliper. Tumor volume was calculated as [(length $\times$ width $\left.^{2}\right) / 2$ ]. On day 18 for DU-145 and on the day 15 for PC-3, a single dose of the GLV- 1 h68 virus $\left(5 \times 10^{6}\right.$ plaque forming units (pfu) in $100 \mu \mathrm{L}$ PBS) was injected into the tail vein (i.v.). The animals of the control groups were injected i.v. with PBS only.

The significance of the results was calculated by two-way analysis of variance (ANOVA) with Bonferroni comparison posttest (GraphPad Prism software, San Diego, USA). The posttest was only performed when ANOVA revealed significance. Results are displayed as means \pm s.d. $P$-values of $<0.05$ were considered significant.

All animal experiments were approved by the government of Unterfranken, Germany, and conducted according to the German animal protection guidelines.

2.6. Histology of Tumors and Lymph Nodes. Preparation of tissue sectioning and fluorescence microscopy was carried out as described previously $[10,18]$. GLV-1h68 was labeled using polyclonal rabbit antivaccinia virus (anti-VACV) antibody (Abcam, Cambridge, UK), which was stained using Cy3-conjugated donkey antirabbit secondary antibodies obtained from Jackson ImmunoResearch (West Grove, PA, USA). Phalloidin-TRITC (Sigma, Taufkirchen, Germany) was used to label actin.

MHCII-positive cells were labeled using monoclonal rat anti-MHCII antibodies (NatuTec, Frankfurt, Germany) and $\mathrm{Cy} 3$-conjugated donkey antirat secondary antibodies (Jackson ImmunoResearch, PA, USA).

The fluorescence-labeled preparations were examined using a Leica MZ 16 FA stereofluorescence microscope equipped with Leica DC500 Digital Camera. Digital images were processed with Photoshop 7.0 (Adobe Systems, Mountain View, CA, USA) and merged to yield pseudocolored images.

2.7. Reverse Transcription (RT)-PCR. Tumor tissue samples were analyzed for the presence of PC-3 cells by RT-PCR. For this purpose, regional lymph nodes with a diameter of more than $2.5 \mathrm{~mm}$ or PC-3 primary tumors were homogenized in TRIzol Reagent (Invitrogen Life Technologies, Karlsruhe, Germany) to isolate total RNA. Genomic DNA was removed from the samples using DNA-free (Ambion, Austin, TX, 

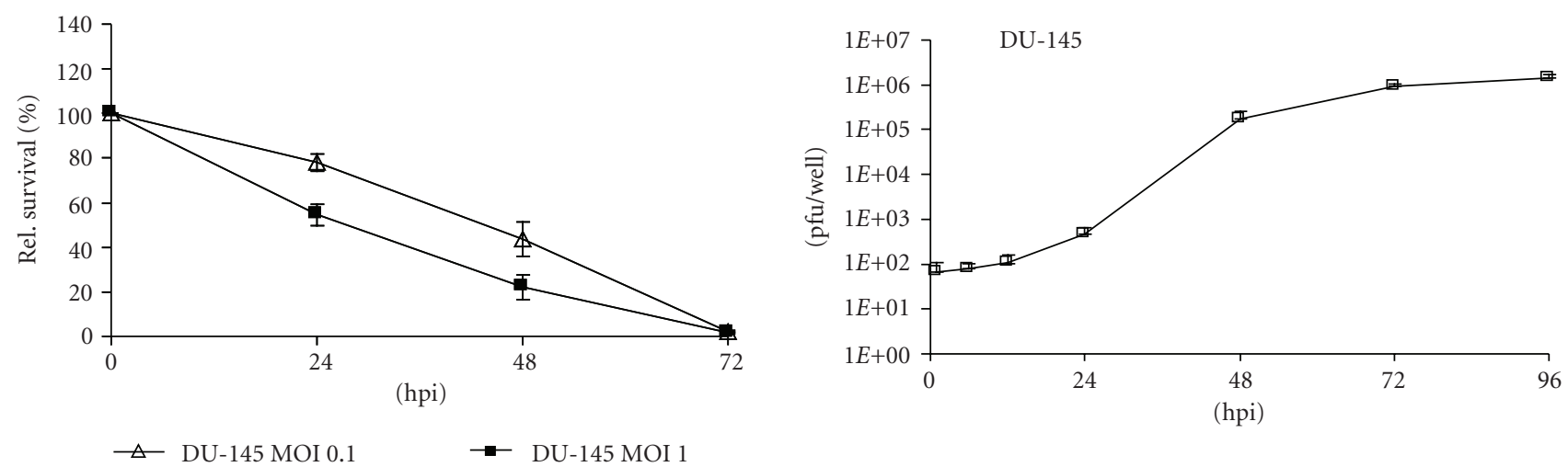

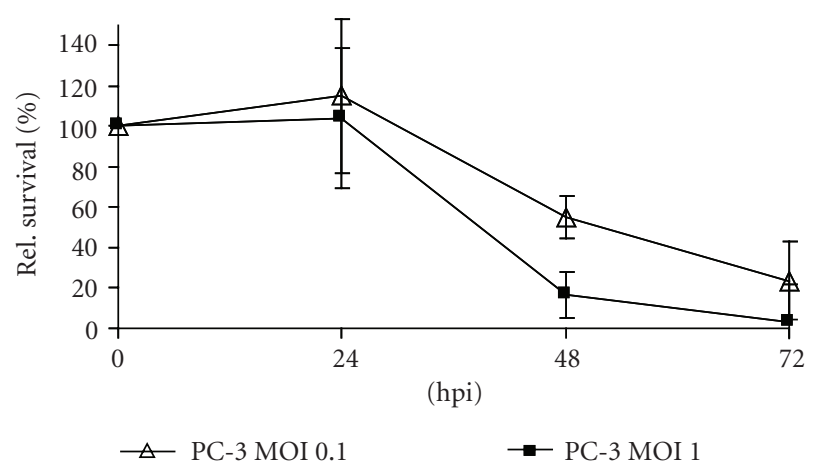

(a)

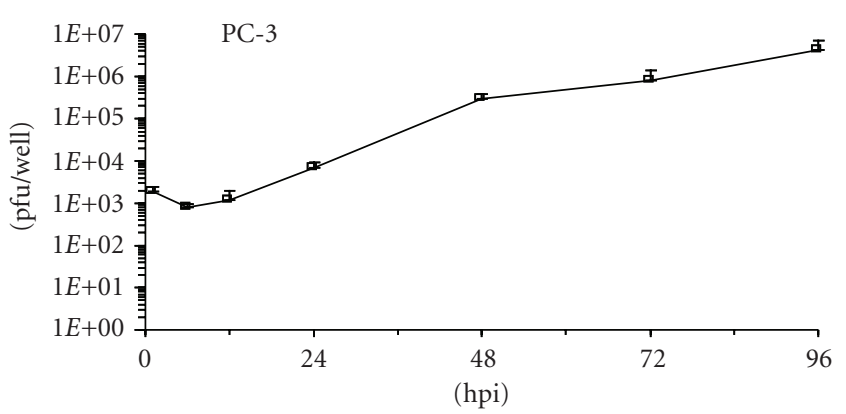

(b)

FIGURE 1: Cytotoxicity (a) and replication (b) of GLV-1h68 virus in human prostate DU-145 and PC-3 cells. (a) Viability of DU-145 and PC3 cells after GLV-1h68 infection at MOIs of 0.1 and 1, respectively, monitored over 72 hours. Values are shown as percentages of respective uninfected controls. (b) Viral titer analysis in DU-145 and PC-3 cell cultures after infection with GLV-1h68 at an MOI of 0.1. Supernatants were collected from virally treated cells at various time points postinfection (hpi). Viral titers were determined as pfu per well in triplicate by plaque assay in CV-1 cell monolayers. Average plus standard deviation are plotted.

USA). RNA samples were converted to cDNA by RevertAid First Strand cDNA Synthesis Kit (Fermentas, St. Leon-Rot, Germany) and analyzed by RT-PCR, using DNA Polymerase (Phusion, Finnzymes, Espoo, Finland) and the primers for human $\beta$-actin (for the identification of PC-3 cells, forward: $5^{\prime}$-CCT CTC CCA AGT CCA CAC AG-3' and reverse: $5^{\prime}$ CTG CCT CCA CCC ACT C- $3^{\prime}$ ); for murine $\beta$-actin (as positive control, forward: $5^{\prime}$-CGT CCA TGC CCT GAG TC$3^{\prime}$ and reverse: $5^{\prime}$-GCT GCC TCA ACA CCT CAA C-3'). PC-3 positive samples were also analyzed for the presence of GLV-1h68 using vaccinia virus A21L specific primers (forward: $5^{\prime}$-CGT AAA CTA CAA ACG TCT AAA CAA GAA- $3^{\prime}$ and reverse: $5^{\prime}$-CCT GGT ATA TCG TCT CTA TCT TTA TCA C-3').

The PCR reaction was run in a T-Gradient Thermoblock PCR machine (Biometra, Göttingen, Germany) under the following cycling conditions: 30 cycles of $95^{\circ} \mathrm{C} / 30 \mathrm{sec}$, $58^{\circ} \mathrm{C} / 30 \mathrm{sec}$, and $72^{\circ} \mathrm{C} / 5 \mathrm{sec}$.

2.8. Preparation of Tumor Lysates for a Mouse ImmuneRelated Protein Antigen Profiling. For preparation of tumor lysates, at 42 days after virus treatment, two mice from each group were sacrificed. Tumors were removed, resuspended in 9 volumes $(\mathrm{W} / \mathrm{V})$ lysis buffer $[50 \mathrm{mM}$ Tris- $\mathrm{HCl}(\mathrm{pH}$ 7.4), $2 \mathrm{mM}$ EDTA (pH 7.4), $2 \mathrm{mM}$ PMSF and Complete Mini protease inhibitors (Roche, Mannheim, Germany)], and lysed using FastPrep FP120 Cell Disruptor (BIO 101, Qbiogene, Germany) at a speed of 6 for 20 seconds (three times). Samples were then centrifuged at $20,000 \mathrm{~g}$ at $4^{\circ} \mathrm{C}$ for 5 minutes and the supernatants were then analyzed for mouse immune-related protein antigen profiling by Multianalyte Profiles (mouse MAPs; Rules Based Medicine, Austin, USA) using antibody linked beads. Results were normalized based on total protein concentration.

\section{Results}

3.1. Cytotoxicity and Replication Efficacy of GLV-1h68 Virus in Human Prostate Cancer DU-145 and PC-3 Cells. In order to test the ability of the GLV-1h68 virus to infect and lyse DU-145 and PC-3 cells, we performed a cell viability assay as described in Materials and Methods. Seventy-two hours after GLV-1h68 infection at MOIs of 0.1 and 1.0, only $1.7 \%$ and $2.1 \%$ of the DU- 145 cells and $23 \%$ and $3 \%$ of the PC-3 cells survived the treatment (Figure 1(a)). 


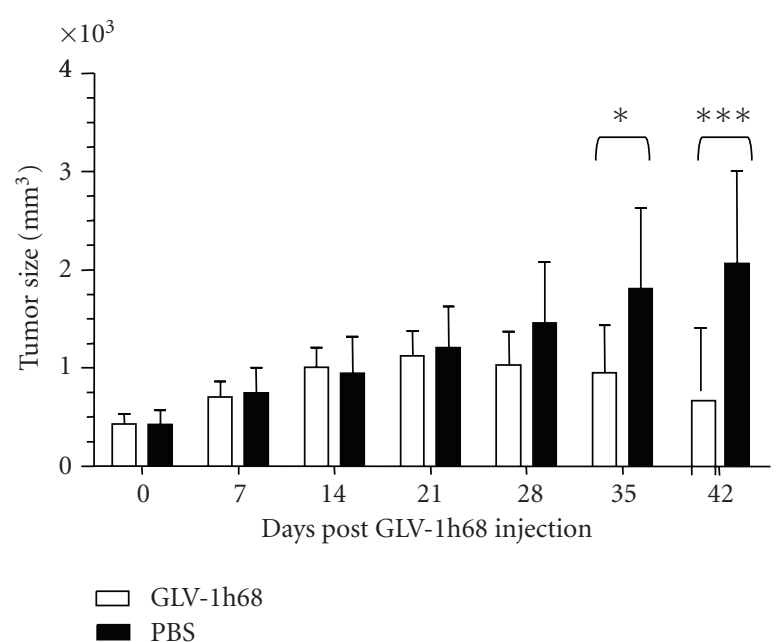

(a)

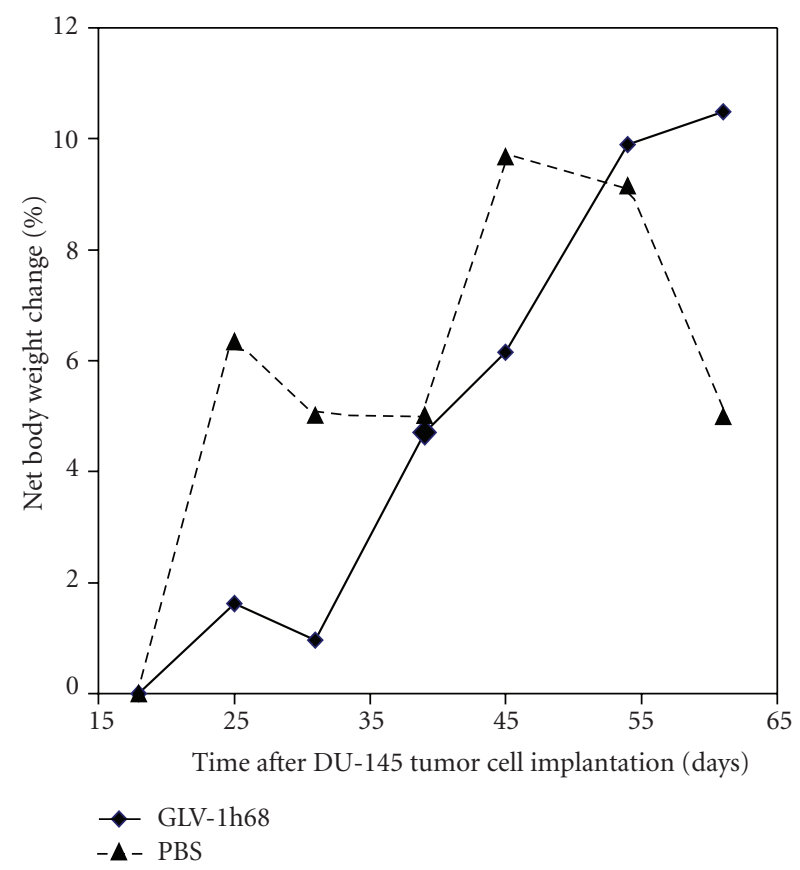

(b)

Figure 2: Effect of systemic virus injection on DU-145 xenograft tumors. (a) DU-145 tumor development in mice after GLV-1h68treatment versus PBS treatment. Two-way analysis of variance (ANOVA) with Bonferroni posttest was used to compare the two corresponding data points of the two groups. $P<.05$ was considered as statistically significant ${ }^{*} P<.05$; ${ }^{* *} P<.01$; ${ }^{* * *} P<$ .001. (b) Body weights of DU-145 cell xenografted mice after virus treatment.

In addition, the replication efficacy of GLV-1h68 in DU145 and PC-3 cells was analyzed in cell culture (Figure 1(b)). The data demonstrated that GLV-1h68 can efficiently replicate in both prostate cancer cell lines and that the highest virus titer was identified in supernatants at 96 hours after infection $\left(1.4 \times 10^{6} \mathrm{pfu} /\right.$ well DU-145 and $2.94 \times 10^{6} \mathrm{pfu} /$ well $\mathrm{PC}-3$, resp.). This result correlated very well with cell viability

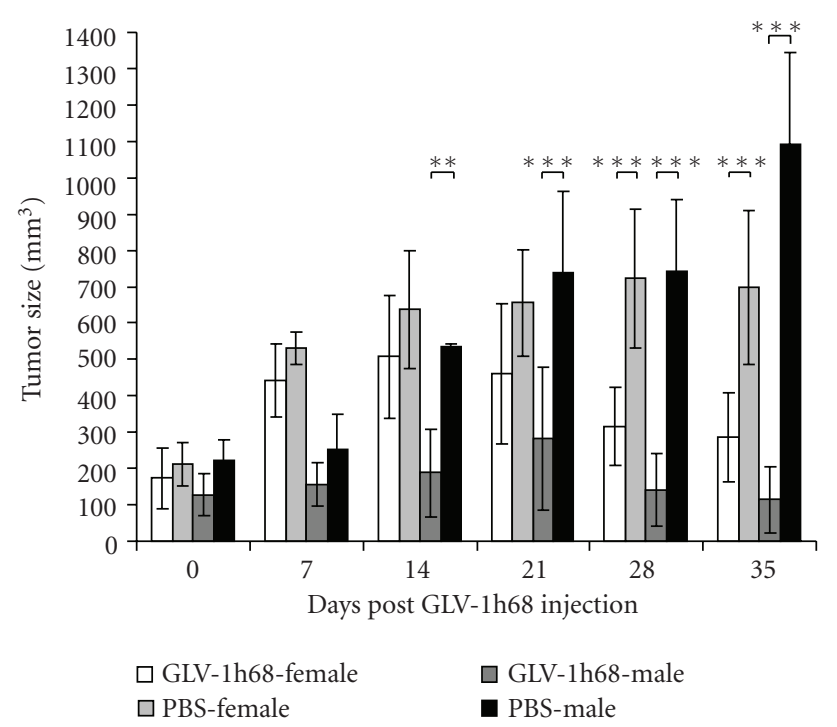

FIGURE 3: Effect of systemic virus injection on tumor growth in PC3 xenografted mice of different genders. Tumor-bearing mice were i.v. injected with either a single dose of GLV- 1 h68 virus $\left(5 \times 10^{6} \mathrm{pfu}\right.$, groups no. 1 and no. 3 ) or with $100 \mu L$ PBS (groups no. 2 and no. 4 ). Tumor volume was monitored weekly. Two-way analysis of variance (ANOVA) with Bonferroni posttest was used to compare the two corresponding data points of the two groups. $P<.05$ was considered as statistically significant ${ }^{*} P<.05 ;{ }^{* *} P<.01$; ${ }^{* *} P<.001$. Male mice: groups no. 1 and no. 2 , female mice groups no. 3 and no. 4 .

assay data (Figure 1(a)). Generally, the GLV-1h68 virus was able to infect, replicate in, and lyse human prostate carcinoma DU-145 and PC-3 cells in cell culture.

3.2. Significant Regression of DU-145 and PC-3 Derived Tumors after a Single Systemic Administration of GLV-1h68. To test the therapeutic capacity of GLV-1h68 against human prostate cancer cells in vivo, nude mice at the age of 6-8 weeks were implanted with either DU-145 or PC-3 cells.

In the case of DU-145 xenografts, at day 18 post infection, ten tumor-bearing male mice were injected with $5 \times 10^{6}$ pfu of GLV-1h68 (Figure 2, group $1, n=6$ ) or with PBS only (Figure 2(a), group 2, $n=4$ ). The data revealed that an intravenous injection of GLV-1h68 significantly inhibited the growth of DU-145 cell xenografts in vivo, while no reduction of net body weight of the animals was observed (Figure 2(b)).

In the case of PC-3 xenografts, two weeks postimplantation, both male and female nude mice developed tumors ranging from 150 to $250 \mathrm{~mm}^{3}$ in size (Figure 3 ). At day 15, five female or male tumor-bearing mice (groups 1 and 3, $n=5$ ) were injected with $5 \times 10^{6}$ pfu of GLV-1h68 into the tail vein. The tumor-bearing mice of control groups 2 (PBSfemale, $n=5$ ) and 4 (PBS-male, $n=5$ ) were injected with PBS only. Tumor size was measured weekly. The data showed that a single GLV-1h68 infection caused highly significant PC-3-tumor regression in both female and male tumorbearing animals (Figure 3, groups 1 and 3), compared to the uninfected control groups 2 and 4 . 

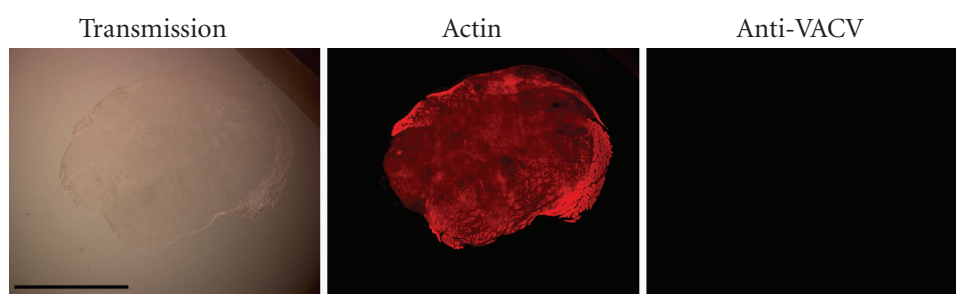

(a)
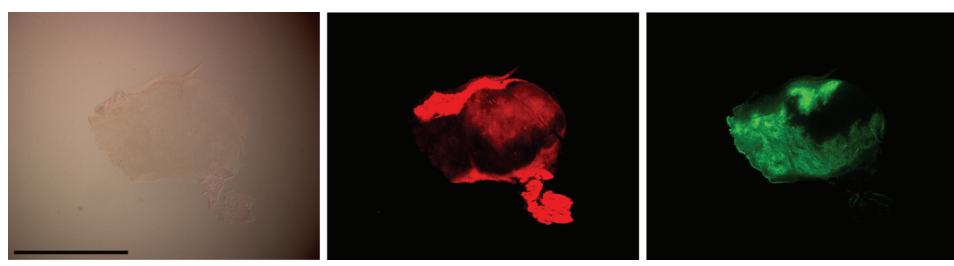

(b)
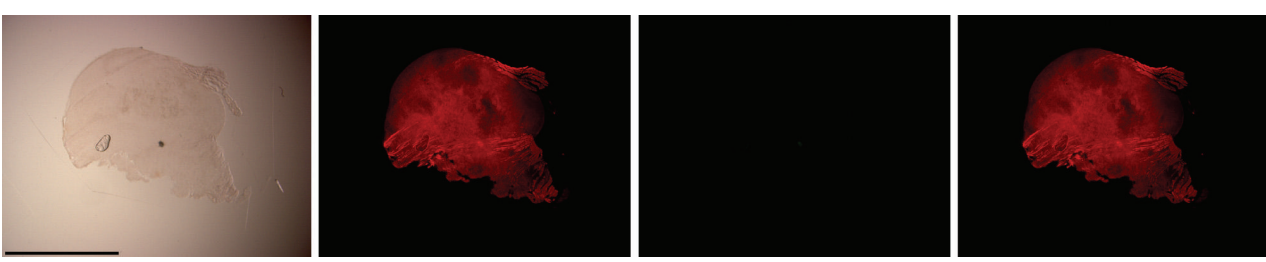

(c)
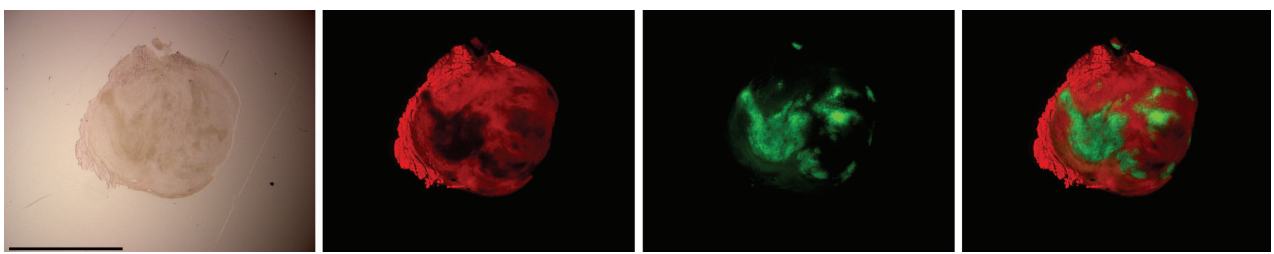

(d)
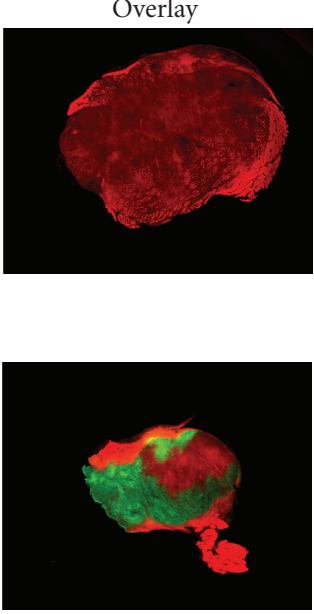

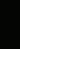




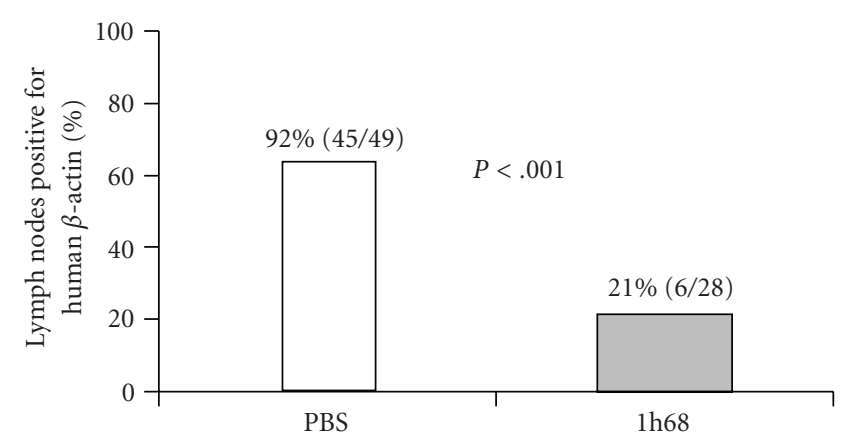

(a)

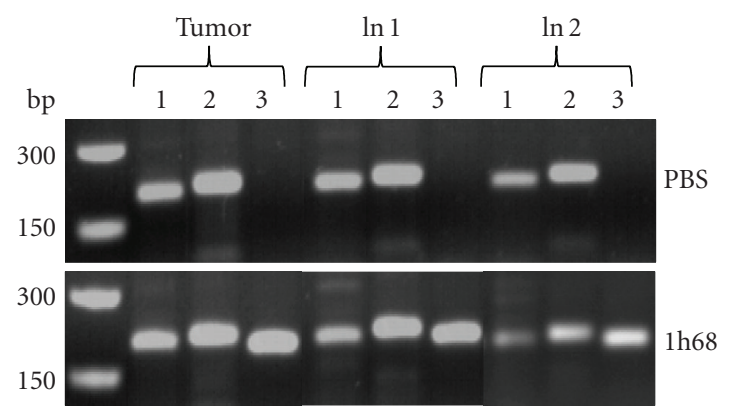

(b)

FIgURE 5: Effects of the GLV-1h68 virus on metastases formation in the regional lymph nodes. (a) Percentage of $\mathrm{PC}-3$ positive regional lymph nodes in tumor-bearing mice at day 21 after PBS or virus injection. Fisher's exact test (GraphPad Prism software, San Diego, USA) was used for statistical analysis. The value of $P<.001$ was considered significant. GLV-1h68:1h68. (b) RT-PCR analysis of tumors and regional lymph nodes of PC-3 xenograft mice injected either with PBS or with $5 \times 10^{6}$ pfu of GLV-1h68 (1h68) using primers for human beta-actin (lines 1; indicating PC-3 cancer cells, PCR-fragment 205 bp), mouse beta-actin (lines 2; positive control, PCR-fragment $216 \mathrm{bp}$ ), and vaccinia virus (lines 3; PCR-fragment 204 bp). ln: lymph node of two different mice.

in control mice $(n=11)$ and $21 \%$ of enlarged lymph nodes (6 of 28 ) in GLV-1h68-treated mice $(n=11)$ were positive for human beta-actin (Figure 5(a)). The presence of human beta-actin was used as a marker for the presence of intact human PC-3 cells; therefore, the systemic treatment by GLV1 h68 appears to affect the PC-3 metastases formation in the regional lymph nodes. All lymph nodes that tested positively for the presence of human beta-actin were also positive for vaccinia virus in GLV-1h68-injected mice but not in control mice (Figure 5(b)), indicating that i.v. injected GLV-1h68 can target lymph node metastases. Interestingly, the GLV-1h68 virus was detected in about $68 \%$ (19 of 28) of the lymph nodes in the absence of PC-3 tumor cells.

3.5. Colonization of Established Metastases in the Regional Lymph Nodes of PC-3 Xenograft Mice. In order to clarify whether the increased inhibition of PC-3 metastases formation in the regional lymph nodes was a result of the preventative or oncolytic effect of the GLV-1h68 virus, we injected tumor-bearing nude mice $(n=4)$ with a single dose of GLV-1h68 $\left(5 \times 10^{6} \mathrm{pfu}\right)$ at day 57 after implantation of PC- 3 cells. This time point allows the analysis of established metastases in the regional lymph nodes of PC-3 xenograft mice. Eleven days later, virus distribution in lymph node metastases was analyzed either by fluorescence imaging (Figure 6) or by immunohistochemical staining (Figure 7). The data demonstrated that GLV-1h68 can efficiently target local lymph node metastases (Figure 6) and destruct tumor tissue within these lymph nodes (Figure 7).

3.6. Mouse Immune-Related Protein Antigen Profiling of GLV1h68-Infected and Noninfected PC-3 Derived Tumors. In order to study the immunological effect of virus infection in vivo, we analyzed the antigen profiling of GLV-1h68infected and noninfected PC-3 tumors 42 days after virus injection. For this purpose, lysates of these tumors were used for examination of the expression levels of immune-related protein antigens of mouse origin, as described in Materials and Methods.

The data revealed that GLV-1h68 injection led to increased production of most of the tested proinflammatory cytokines and chemokines (Table 2). Many of the tested cytokines and chemokines, such as IP-10, IL-18, IL-6, MCP1, MCP-3, MCP-5, M-CSF, and TNF-alpha, are known to activate macrophages, monocytes, neutrophils, eosinophils, and, so forth, and to trigger proinflammatory responses in target tissues. In contrast, only one cytokine (MIP-1-gamma) was significantly downregulated upon virus infection in both male and female PC-3 tumor-bearing mice.

3.7. Active Peri- and Intra-Tumoral Infiltration of MHC Class II-Expressing Host Cells in GLV-1h68-Infected Tumors. The immune-related protein antigen profiling of regressing PC3 -tumors revealed protein expression signatures consistent with innate immune defense activation (Table 2). Therefore, we analyzed the presence of innate immune system components into the PC-3-derivated tumors by immunohistochemistry. In this context, we labelled MHC class IIexpressing host cells (like, e.g., macrophages and dendritic cells) in the tumor tissue. Indeed, the data demonstrated specific peri- and intra-tumoral infiltration of MHC class IIexpressing host cells surrounding virus-infected cancer cells (Figure 8). These host cells were absent in the noninfected control samples.

\section{Discussion}

Prostate cancer is the cause of more than $1 \%$ of all deaths in men and its incidence is increasing by $2-3 \%$ per year [19]. The available treatment options for advanced prostate cancer are very limited and the prognosis for patients with advanced-stage disease is poor. Therefore, there is an urgent need to identify novel agents for therapy and diagnosis of advanced prostate cancer.

In this study, we investigated the therapeutic efficacy of the oncolytic vaccinia virus strain GLV-1h68 against advanced human prostate carcinoma. We examined the antitumor efficacy of GLV-1h68 in DU-145 and PC-3 prostate 

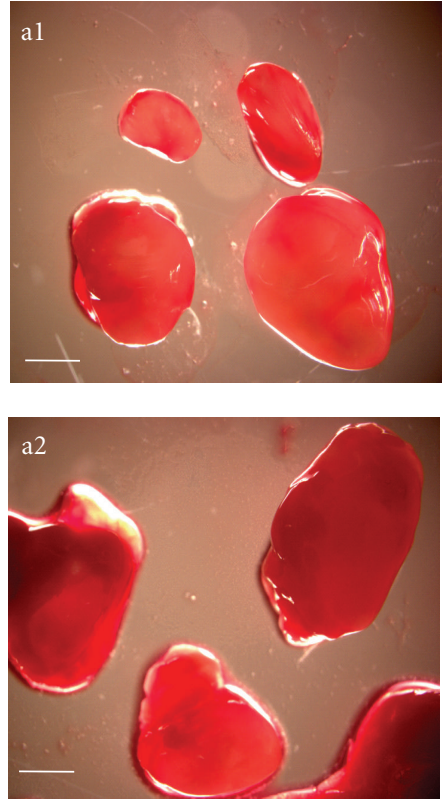

(a)
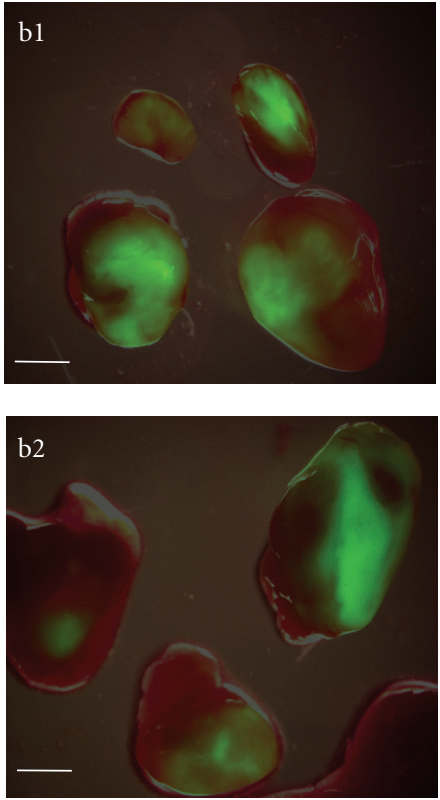

(b)

FIGURE 6: Detection of lymph node metastases by GLV-1h68-mediated fluorescence. (a) Bright-field, and (b) overlay fluorescence images of renal and lumbar lymph nodes in GLV-1h68-infected mice with PC-3 tumors. a1, b1: mouse no. 1 and a2, b2: mouse no. 2 . Scale bars = $2 \mathrm{~mm}$.

tumor xenograft models. The androgen-independent DU145 and PC-3 models were preferred over several androgendependent xenograft models, like LAPC-4 or $-9[20,21]$, since the PC-3 cells are more aggressive [22] and more drugresistant than that used in androgen-dependent models [23]. It is known that the mortality of prostate cancer results from the progression of androgen-dependent disease to androgenindependent prostatic growth and metastases to the bones and lymph nodes $[24,25]$. Therefore, we also analyzed the presence of PC3-metastases in the regional lymph nodes. The tumor status of these lymph nodes provides important information in both the diagnosis and treatment of prostate cancer. Our PC-3 xenografts data demonstrated that GLV1 h68 can efficiently target both the primary tumor and the regional lymph nodes with metastases. These interactions led to significant size reduction of the primary tumor and inhibition of metastases development in our PC-3 xenograft models.

In order to test the effect of GLV-1h68 on PC-3 metastases formation, we analyzed the presence of PC-3 cells in the regional lymph nodes at day 21 after PBS or GLV1 h68 injection. We chose this time point because, at 21 days after the virus treatment, the growth of the primary tumors was efficiently inhibited (Figure 3 ). We found a significant reduction in the number of PC-3 positive lymph nodes in virus-treated tumor-bearing mice compared to non-virus-treated controls (Figure 5(a)). Moreover, lymph nodes bearing PC-3 cells in GLV-1h68-injected animals showed also the presence of vaccinia virus (Figure 5(b)). In addition, histological analysis revealed virus patches over the surface of metastases, which was also associated with massive destruction of tumor tissue when GLV-1h68 was injected into mice with well-established PC-3 lymph node metastases (Figure 7). Surprisingly, the GLV-1h68 virus was detected in about $68 \%$ lymph nodes in the absence of PC-3 actin data. In these cases, it could be that, the PC-3 cells were already destroyed, but GLV-1h68 persisted longer in the tumor tissue or the vaccinia virus was present in lymph nodes that were not infiltrated by malignant cells. Therefore, the significantly reduced number of lymph node metastases in GLV-1h68injected PC-3 mice could be due to both the oncolytic effects of vaccinia virus colonizing lymph node metastases and the virusprevention of metastases formation.

For a better understanding of a possible immunological effect of the GLV-1h68 virus on PC-3 xenografts, we investigated the mouse immune-related protein antigen profiling in the primary tumors with and without virus infection. The data revealed that the protein expression levels of most of the tested proinflammatory cytokines and chemokines were significantly upregulated after viral tumor colonization (Table 2). The resulting cytokine profile after GLV-1h68 infection was similar to the cytokine profile in immunocompetent Balb/c mice $[14,26]$ and in an immunocompromised nude mouse model [11]. Therefore, the cytokine profile after vaccinia virus infection seems to be relatively independent of several factors, including: tumor origin [[11] and this study], the number of replication-competent virus particles reaching the tumor after injection, the route of injection [[10], Gentschev unpublished data)], and the sex of the mice (this study). These observations support the hypothesis that the oncolytic effect of vaccinia virus is at least in part mediated through host defense mechanisms, most likely 


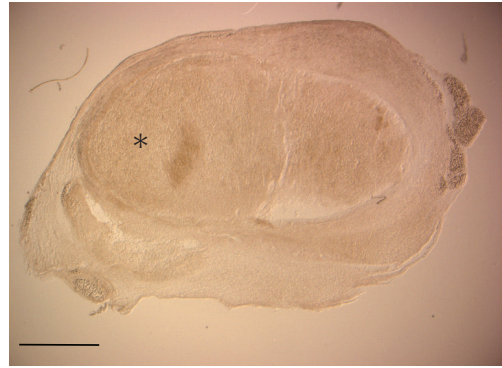

(a)

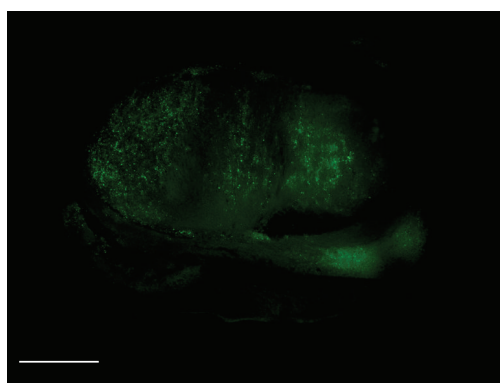

(c)

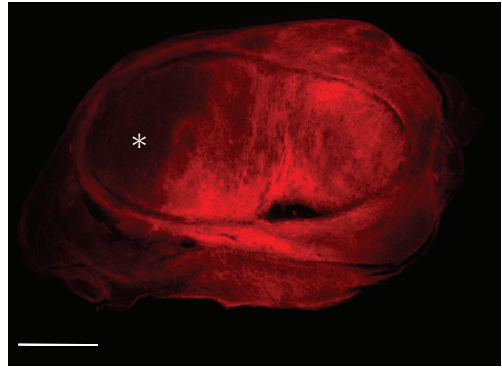

(b)

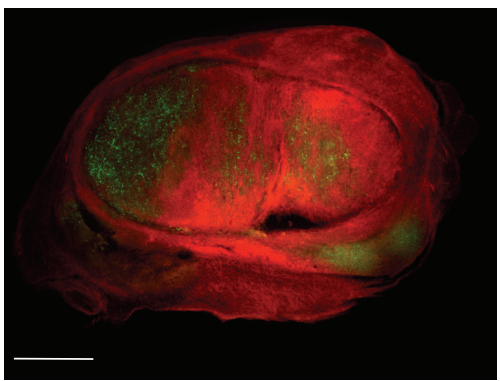

(d)

FIGURE 7: Immunohistochemical staining of a lymph node with PC-3 metastases. At day 57 after PC-3 cells implantation, tumor-bearing mice were injected with $5 \times 10^{6}$ pfu of GLV-1h68. At day 11 after virus injection, whole lumbar lymph node cross-sections were analyzed by bright-field imaging (a) or by fluorescence microscopy techniques. (b) Phalloidin-TRITC (red) and (c) anti-VACV antibody (green), and (d) (overlay). Scale bars $=1 \mathrm{~mm}$. *indicates the dead tumor tissue destructed by GLV-1h68.
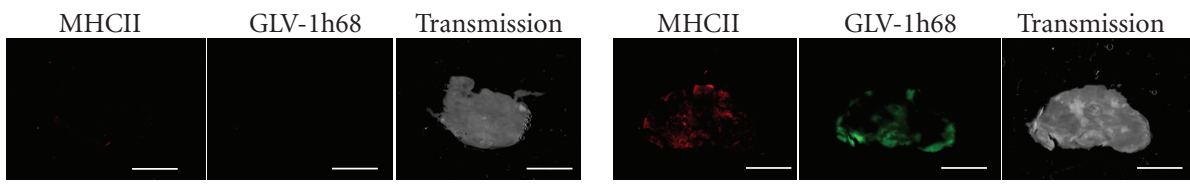

(a)
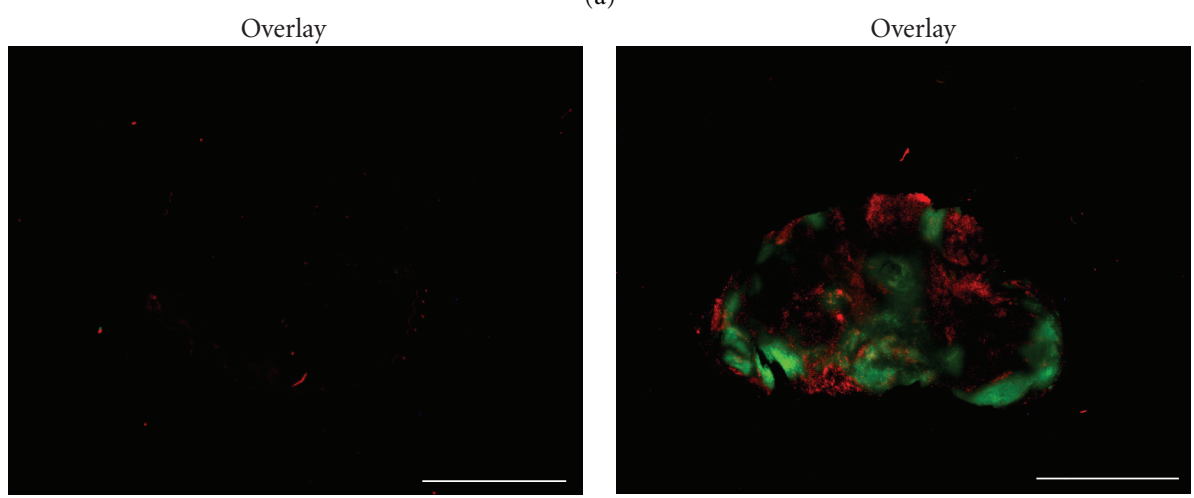

(b)

FIGURE 8: Immunohistochemistry staining of MHC class II positive cells in PC-3 tumors. (a) Forty-two days after GLV-1h68 administration PC-3 tumors were excised, sectioned, and labeled for MHCII. In addition, GFP signals from GLV-1h68-infected cells and transmission images are shown. (b) Overlay of MHCII and GFP signals in uninfected (left) and virus-infected PC-3 tumors (right). Scale bars $=5 \mathrm{~mm}$.

by the innate immune system $[8,27]$. Indeed, many of the upregulated proteins in tumor tissue (Table 2), such as MCP-1, MCP-3, MCP-5, M-CSF, and IL-18, are involved in activation of some components of the innate immune system, like macrophages and neutrophils. IP-10, the most upregulated protein in our study, has been shown to display antitumor and antimetastatic properties [28]. This cytokine is also known to enhance the cytolytic activity of NK cells, a 
TABLE 2: Mouse immune-related protein antigen profiling in PC-3-derived tumors of male or female nude mice with or without GLV-1h68 treatment at the day 42 after virus infection $(n=2)$. Folds of enhancement (a) or suppression (b) of mouse protein expression after virus injection are shown. Each value was determined as the mean of triplicate determination.

(a) Upregulated protein expression levels (day 42 after virus infection).

\begin{tabular}{lccl}
\hline Antigen names & GLV-1h68/untreated ratio (female) & GLV-1h68/untreated ratio (male) & Classification \\
\hline Apo A1 & 2.83 & 2.78 & $\begin{array}{l}\text { Anti-inflammatory protein } \\
\text { Granulocyte-macrophage } \\
\text { colony-stimulating factor }\end{array}$ \\
GM-CSF & 2.2 & 2.77 & Proinflammatory cytokine \\
IL-6 & 8.44 & 6.8 & Pleiotropic cytokine \\
IL-11 & 3.81 & 3.63 & Proinflammatory cytokine \\
IL-18 & 30 & 29.72 & $\begin{array}{l}\text { Interferon-gamma-induced } \\
\text { protein }\end{array}$ \\
IP-10 (CXCL10) & 33.91 & 8.18 & Proinflammatory cytokine \\
MCP-1 (CCL2) & 11.8 & 7.05 & Proinflammatory cytokine \\
MCP-3 (CCL7) & 6.09 & 3.23 & Proinflammatory cytokine \\
MCP-5 (CCL12) & 30.24 & 10.64 & Proinflammatory cytokine \\
M-CSF (KC/GRO $\alpha)$ & 2.18 & 2.48 & $\begin{array}{l}\text { Proinflammatory } \\
\text { chemokine }\end{array}$ \\
MDC (CCL22) & 4.5 & 3.23 & Proinflammatory cytokine \\
MIP-1beta & 9.09 & 3.31 & $\begin{array}{l}\text { Proinflammatory } \\
\text { chemokine }\end{array}$ \\
MIP-2 (CXCL2) & 2.9 & 3.33 & $\begin{array}{l}\text { Proinflammatory } \\
\text { chemokine }\end{array}$ \\
RANTES (CCL5) & 7.1 & 4.58 & Tissue inhibitor of \\
metalloproteinase type-1 & Proinflammatory cytokine \\
TIMP-1 & 2.6 & 2.43 &
\end{tabular}

(b) Downregulated protein expression level (day 42 after virus infection).

\begin{tabular}{lccc}
\hline Antigen names & Untreated GLV-1h68/ratio (female) & untreated GLV-1h68/ratio (male) & Classification \\
\hline MIP-1gamma (CCL9) & 8 & 7.5 & Cytokine \\
\hline
\end{tabular}

component of the innate immune system, and to contribute to vaccinia virus clearance in vivo [29].

The only cytokine in our study that was significantly downregulated was the protein MIP-1 gamma (CCL9). MIP1 gamma, a small cytokine belonging to the CC chemokine family, is secreted by the follicle-associated epithelium and recruits $\mathrm{CD} 11 \mathrm{~b}+$ dendritic cells [30]. Interestingly, in some adenocarcinomas, the expression of MIP-1 gamma is increased in the tumor epithelium [31]. However, the effect of the down-regulation of MIP-1 gamma in our PC3 prostate tumor xenograft models is currently unknown.

Taken together, the immune-related protein antigen profiling of regressing PC-3 tumors revealed protein expression signatures consistent with innate immune defense activation inclusive of cytokines and chemokines (Table 2). Therefore, we analyzed the presence of some components of the innate immune system such as macrophages, dendritic cells, and leukocytes into the PC-3-derivated tumors by immunohistochemistry. The data demonstrated specific peri- and intratumoral infiltration of MHC class II-expressing host cells surrounding virus-infected cancer cells (Figure 8). The presence of activated macrophages or dendritic cells in virusinfected PC-3 xenografts only could serve as an evidence for the association between xenograft eradication and immune activation of the innate immune system. These findings suggest that immune activation may combine with viral oncolysis to induce tumor eradication, providing a novel perspective for the design of oncolytic viral therapies for human cancers. However, in our experimental settings, it was not possible to distinguish between components of the innate immune system directly involved in the killing of tumor cells or in the elimination of vaccinia virus particles.

As described previously, we have used male and female nude mice for analysis of the effect of the GLV-1h68 virus on PC-3 xenografts. The data demonstrated that in male mice, GLV-1h68 injection led to a faster and more efficient tumor regression than in female mice bearing PC-3 tumors. However, significant tumor regression was found in both male and female PC-3 xenografts at day 28 after virus injection. In addition, we did not find any significant differences 
in the resulting cytokine profiles between male and female PC-3 xenografts after virus injection (Table 2). This means that female nude mice could also be successfully used in experiments with PC-3 xenografts. This is an advantage, since the experimental handling of female animals is easier than the handling of male animals.

In summary, the GLV-1h68 strain showed an outstanding antitumor effect and a safety profile in DU-145 and PC3 -xenografts. This makes it a promising candidate for the treatment of advanced metastatic prostate cancer in human.

\section{Conclusion}

Our data demonstrate that the oncolytic virus GLV-1h68 can efficiently infect and destroy the human prostate DU-145 and PC-3 cells in cell culture and in vivo. In addition, a single systemic administration of GLV-1h68 causes a significant inhibition of the growth of primary tumor and metastases in PC-3 xenografts. Our findings suggest that the vaccinia virus GLV-1h68 has the potential to treat prostate cancer in human patients.

\section{Acknowledgments}

The authors declare that they have competing interests. I. Gentschev, Y. A. Yu, N. Chen, J. Stritzker, and A. A. Szalay have financial interests in Genelux Corporation. E. Hofmann, U. Donat, M. Adelfinger, V. Raab, and S. Weibel were supported by grants of Genelux Corporation. The costs of publication of this paper were defrayed in part by the payment of page charges. This paper must therefore be hereby marked advertisement in accordance with 18 U.S.C. Section 1734 solely to indicate this fact. They thank Ms. J. Langbein, Mr. Jason Aguilar, and Mr. Terry Trevino for excellent technical support, Ms. A. Feathers and Ms. Z. Sokolovic for critical reading of the paper. Ivaylo Gentschev and Ulrike Donat contributed equally to this work.

\section{References}

[1] A. Jemal, R. Siegel, E. Ward, T. Murray, J. Xu, and M. J. Thun, "Cancer statistics, 2007," CA: A Cancer Journal for Clinicians, vol. 57, no. 1, pp. 43-66, 2007.

[2] L. Cheng, H. Zincke, M. L. Blute, E. J. Bergstralh, B. Scherer, and D. G. Bostwick, "Risk of prostate carcinoma death in patients with lymph node metastasis," Cancer, vol. 91, no. 1, pp. 66-73, 2001.

[3] P. R. Carroll, K. L. Lee, Z. Y. Fuks, and P. W. Kantoff, "Cancer of the prostate," in Cancer: "Principals and Practices of Oncology", V. T. DeVita, S. Hellman, and S. A. Rosenberg, Eds., pp. 14181479, Lippincott-Raven, New York, NY, USA, 2001.

[4] R. Cattaneo, T. Miest, E. V. Shashkova, and M. A. Barry, "Reprogrammed viruses as cancer therapeutics: targeted, armed and shielded," Nature Reviews Microbiology, vol. 6, no. 7, pp. 529-540, 2008.

[5] M. J. V. Vähä-Koskela, J. E. Heikkilä, and A. E. Hinkkanen, "Oncolytic viruses in cancer therapy," Cancer Letters, vol. 254, no. 2, pp. 178-216, 2007.

[6] A. M. Crompton and D. H. Kirn, "From ONYX-015 to armed vaccinia viruses: the education and evolution of oncolytic virus development," Current Cancer Drug Targets, vol. 7, no. 2, pp. 133-139, 2007.

[7] T.-C. Liu, E. Galanis, and D. Kirn, "Clinical trial results with oncolytic virotherapy: a century of promise, a decade of progress," Nature Clinical Practice Oncology, vol. 4, no. 2, pp. 101-117, 2007.

[8] Q. Zhang, Y. A. Yu, E. Wang, et al., "Eradication of solid human breast tumors in nude mice with an intravenously injected light-emitting oncolytic vaccinia virus," Cancer Research, vol. 67, no. 20, pp. 10038-10046, 2007.

[9] K. J. Kelly, Y. Woo, P. Brader, et al., "Novel oncolytic agent GLV-1h68 is effective against malignant pleural mesothelioma," Human Gene Therapy, vol. 19, no. 8, pp. 774-782, 2008.

[10] I. Gentschev, J. Stritzker, E. Hofmann, et al., "Use of an oncolytic vaccinia virus for the treatment of canine breast cancer in nude mice: preclinical development of a therapeutic agent," Cancer Gene Therapy, vol. 16, no. 4, pp. 320-328, 2009.

[11] Y. A. Yu, C. Galanis, Y. Woo, et al., "Regression of human pancreatic tumor xenografts in mice after a single systemic injection of recombinant vaccinia virus GLV-1h68," Molecular Cancer Therapeutics, vol. 8, no. 1, pp. 141-151, 2009.

[12] S.-F. Lin, D. L. Price, C.-H. Chen, et al., "Oncolytic vaccinia virotherapy of anaplastic thyroid cancer in vivo," Journal of Clinical Endocrinology and Metabolism, vol. 93, no. 11, pp. 4403-4407, 2008.

[13] S.-F. Lin, Z. Yu, C. Riedl, et al., "Treatment of anaplastic thyroid carcinoma in vitro with a mutant vaccinia virus," Surgery, vol. 142, no. 6, pp. 976-983, 2007.

[14] K. J. Kelly, P. Brader, Y. Woo, et al., "Real-time intraoperative detection of melanoma lymph node metastases using recombinant vaccinia virus GLV-lh68 in an immunocompetent animal model," International Journal of Cancer, vol. 124, no. 4, pp. 911-918, 2009.

[15] N. Chen, Q. Zhang, Y. A. Yu, et al., "A novel recombinant vaccinia virus expressing the human norepinephrine transporter retains oncolytic potential and facilitates deep-tissue imaging," Molecular Medicine, vol. 15, no. 5-6, pp. 144-151, 2009.

[16] P. Brader, K. J. Kelly, N. Chen, et al., "Imaging a genetically engineered oncolytic vaccinia virus (GLV-1h99) using a human norepinephrine transporter reporter gene," Clinical Cancer Research, vol. 15, no. 11, pp. 3791-3801, 2009.

[17] T.-C. Liu and D. Kirn, "Systemic efficacy with oncolytic virus therapeutics: clinical proof-of-concept and future directions," Cancer Research, vol. 67, no. 2, pp. 429-432, 2007.

[18] S. Weibel, J. Stritzker, M. Eck, W. Goebel, and A. A. Szalay, "Colonization of experimental murine breast tumours by Escherichia coli K-12 significantly alters the tumour microenvironment," Cellular Microbiology, vol. 10, no. 6, pp. 12351248, 2008.

[19] N. Salesi, P. Carlini, E. M. Ruggeri, G. Ferretti, E. Bria, and F. Cognetti, "Prostate cancer: the role of hormonal therapy," Journal of Experimental and Clinical Cancer Research, vol. 24, no. 2, pp. 175-180, 2005.

[20] N. Craft, Y. Shostak, M. Carey, and C. L. Sawyers, "A mechanism for hormone-independent prostate cancer through modulation of androgen receptor signaling by the HER-2/neu tyrosine kinase," Nature Medicine, vol. 5, no. 3, pp. 280-285, 1999.

[21] N. Craft, C. Chhor, C. Tran, et al., "Evidence for clonal outgrowth of androgen-independent prostate cancer cells from androgen-dependent tumors through a two-step process," Cancer Research, vol. 59, no. 19, pp. 5030-5036, 1999. 
[22] E. Brakenhielm, J. B. Burton, M. Johnson, et al., "Modulating metastasis by a lymphangiogenic switch in prostate cancer," International Journal of Cancer, vol. 121, no. 10, pp. 21532161, 2007.

[23] S. Mohapatra, B. Chu, X. Zhao, and W. J. Pledger, "Accumulation of p53 and reductions in XIAP abundance promote the apoptosis of prostate cancer cells," Cancer Research, vol. 65, no. 17, pp. 7717-7723, 2005.

[24] B. J. Feldman and D. Feldman, "The development of androgen-independent prostate cancer," Nature Reviews Cancer, vol. 1, no. 1, pp. 34-45, 2001.

[25] E. M. Bruckheimer and N. Kyprianou, "Apoptosis in prostate carcinogenesis: a growth regulator and a therapeutic target," Cell and Tissue Research, vol. 301, no. 1, pp. 153-162, 2000.

[26] C. W. Knorr, S. D. Allen, A. R. Torres, and D. F. Smee, "Effects of cidofovir treatment on cytokine induction in murine models of cowpox and vaccinia virus infection," Antiviral Research, vol. 72, no. 2, pp. 125-133, 2006.

[27] A. Worschech, D. Haddad, D. F. Stroncek, E. Wang, F. M. Marincola, and A. A. Szalay, "The immunologic aspects of poxvirus oncolytic therapy," Cancer Immunology, Immunotherapy, vol. 58, no. 9, pp. 1355-1362, 2009.

[28] A. D. Luster and P. Leder, "IP-10, a -C-X-C- chemokine, elicits a potent thymus-dependent antitumor response in vivo," Journal of Experimental Medicine, vol. 178, no. 3, pp. 10571065, 1993.

[29] S. Mahalingam, J. M. Farber, and G. Karupiah, "The interferon-inducible chemokines MuMig and Crg-2 exhibit antiviral activity in vivo," Journal of Virology, vol. 73, no. 2, pp. 1479-1491, 1999.

[30] X. Zhao, A. Sato, C. S. Dela Cruz, et al., "CCL9 is secreted by the follicle-associated epithelium and recruits dome region Peyer's patch CD11 ${ }^{+}$dendritic cells," Journal of Immunology, vol. 171, no. 6, pp. 2797-2803, 2003.

[31] T. Kitamura, K. Kometani, H. Hashida, et al., "SMAD4deficient intestinal tumors recruit $\mathrm{CCR} 1^{+}$myeloid cells that promote invasion," Nature Genetics, vol. 39, no. 4, pp. 467$475,2007$. 


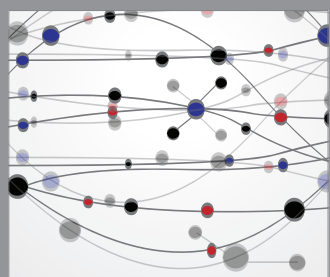

The Scientific World Journal
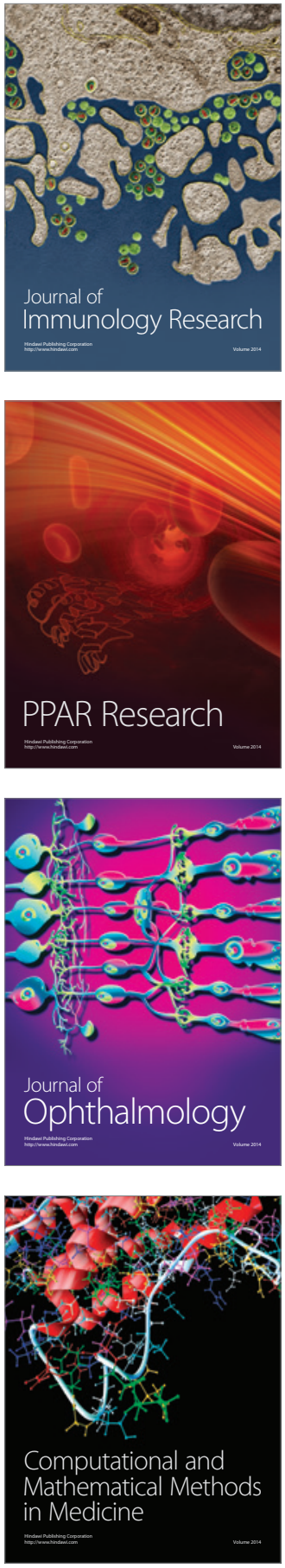

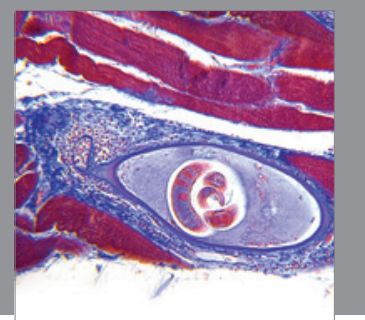

Gastroenterology

Research and Practice
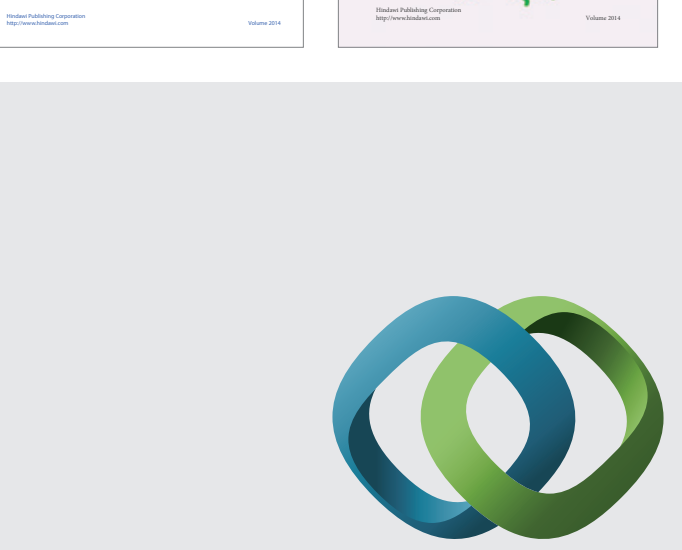

\section{Hindawi}

Submit your manuscripts at

http://www.hindawi.com
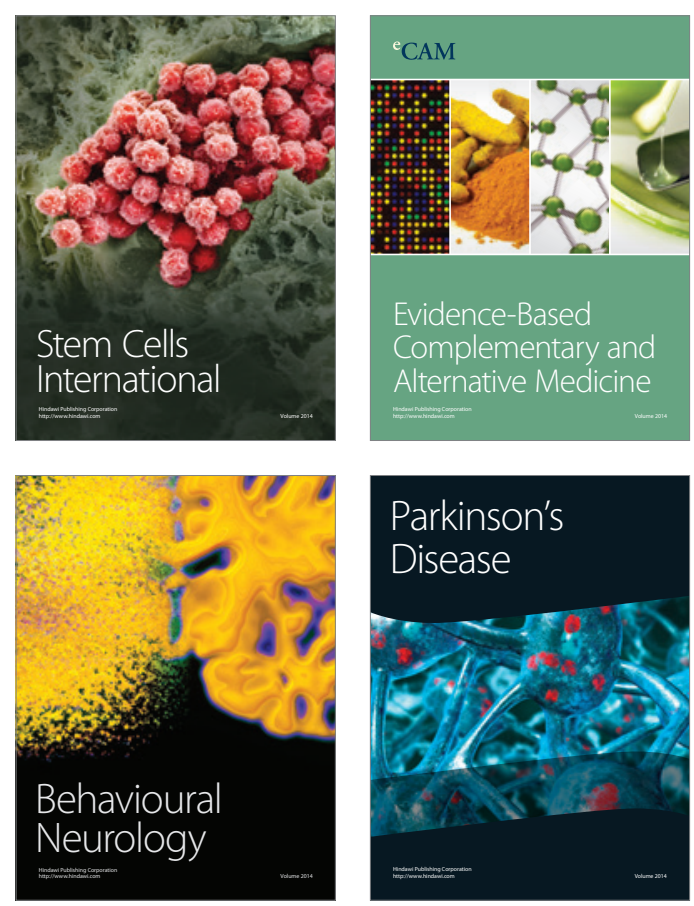

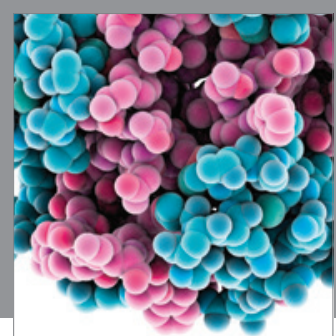

Journal of
Diabetes Research

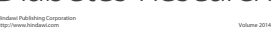

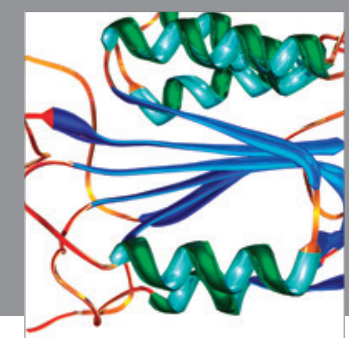

Disease Markers
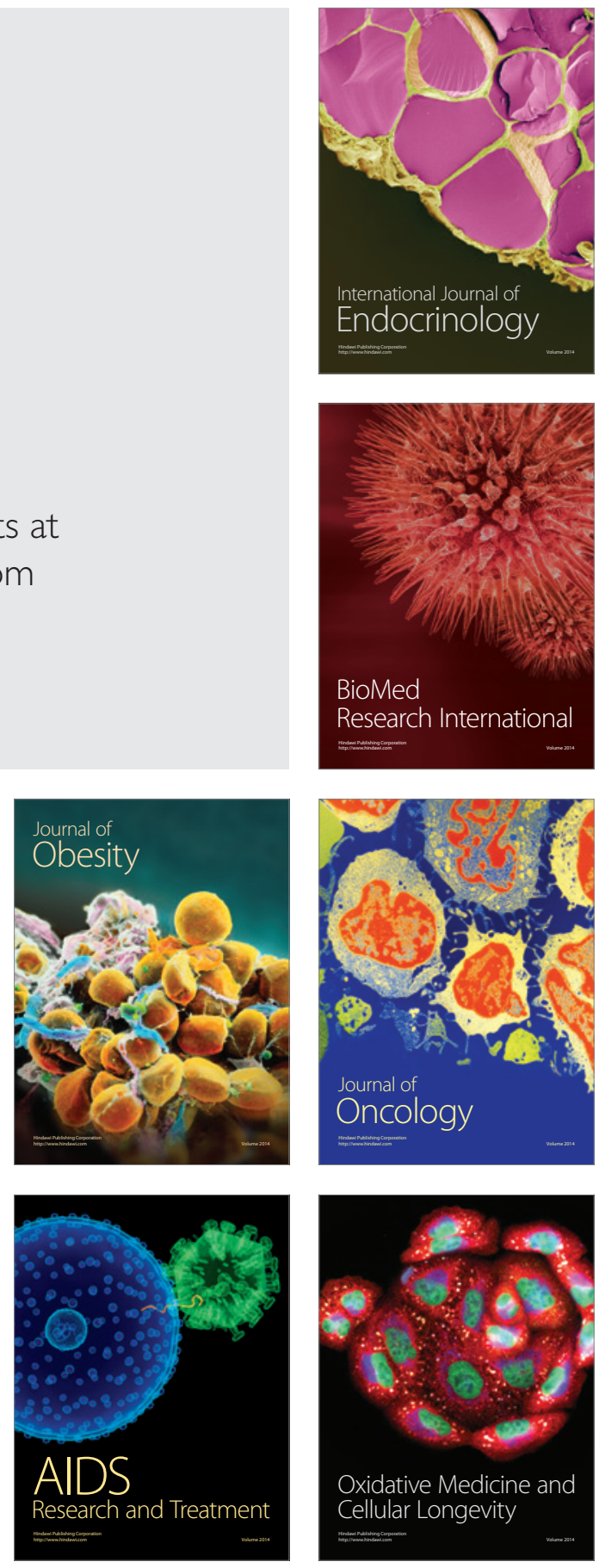